Case Report

\title{
A Case of Perforated Sigmoid Diverticulitis in Which Gram Staining of Ascitic Fluid Was Useful for Diagnosis
}

\author{
Junko Tsuchida, ${ }^{1}$ Shouhei Fujita, ${ }^{1}$ Fumihiro Kawano, ${ }^{1}$ Ryoichi Tsukamoto, ${ }^{1}$ \\ Kunpei Honjo, ${ }^{1}$ Shigetoshi Naito, ${ }^{1}$ Shun Ishiyama, ${ }^{1}$ Shozo Miyano, ${ }^{1}$ \\ Michio Machida, ${ }^{1}$ Toshiaki Kitabatake, ${ }^{1}$ Minoru Fujisawa, ${ }^{1}$ Kuniaki Kojima, \\ Kanako Ogura, ${ }^{2}$ and Toshiharu Matsumoto ${ }^{2}$ \\ ${ }^{1}$ Department of General Surgery, Juntendo University Nerima Hospital, 3-1-10 Takanodai, Nerimaku, Tokyo 177-8521, Japan \\ ${ }^{2}$ Department of Clinical Pathology, Juntendo University Nerima Hospital, 3-1-10 Takanodai, Nerimaku, Tokyo 177-8521, Japan
}

Correspondence should be addressed to Junko Tsuchida; j.tsuchida117@gmail.com

Received 5 December 2013; Accepted 19 January 2014; Published 4 March 2014

Academic Editors: F.-M. Haecker and H. Imura

Copyright (C) 2014 Junko Tsuchida et al. This is an open access article distributed under the Creative Commons Attribution License, which permits unrestricted use, distribution, and reproduction in any medium, provided the original work is properly cited.

\begin{abstract}
An 85-year-old woman was admitted to our hospital for steroid therapy for relapsing nephrotic syndrome. During hospitalization, she complained of sudden epigastric pain at night. Although there were signs of peritoneal irritation, CT showed a large amount of ascitic fluid, but no free intraperitoneal gas. Gram staining of ascitic fluid obtained by abdominal paracentesis showed Gramnegative rods, which raised a strong suspicion of gastrointestinal perforation and peritonitis. Therefore, emergency surgery was performed. Exploration of the colon showed multiple sigmoid diverticula, one of which was perforated. The patient underwent an emergency Hartmann's procedure. Imaging studies failed to reveal any evidence of gastrointestinal perforation, presenting a diagnostic challenge. However, a physician performed rapid Gram staining of ascitic fluid at night when laboratory technicians were absent, had a strong suspicion of gastrointestinal perforation, and performed emergency surgery. Gram staining is superior in rapidity, and ascitic fluid Gram staining can aid in diagnosis, suggesting that it should be actively performed. We report this case, with a review of the literature on the significance of rapid diagnosis by Gram staining.
\end{abstract}

\section{Introduction}

The presence of free intraperitoneal air (or pneumoperitoneum) is an important finding in the diagnosis of gastrointestinal perforation. However, pneumoperitoneum is not always associated with gastrointestinal perforation. In the absence of free intraperitoneal air, whether or not to perform surgery must be determined based on abdominal physical findings or other diagnostic means. In particular, the prognosis of patients with large-bowel perforation reportedly depends on the time to surgery, necessitating rapid diagnosis.

Herein, we report a case of sigmoid colon perforation diagnosed by rapid, very simple Gram staining of ascitic fluid, with a review of the literature.

\section{Case Report}

The patient was an 85-year-old woman with a chief complaint of epigastric pain and a past history of nephrotic syndrome, hyperlipidemia, diabetes mellitus, and status postbilateral cataract surgery. In 2009, she developed nephrotic syndrome, which was treated with steroid therapy until February 2011, when complete remission was achieved and the therapy was discontinued. No kidney biopsy was performed because of her advanced age, and the cause of her nephrotic syndrome remained unknown. In February 2013, the nephrotic syndrome relapsed, and she was admitted to the Department of Nephrology of our hospital for steroid therapy. Steroid semipulse therapy $(500 \mathrm{mg}$ of methylprednisolone daily for 


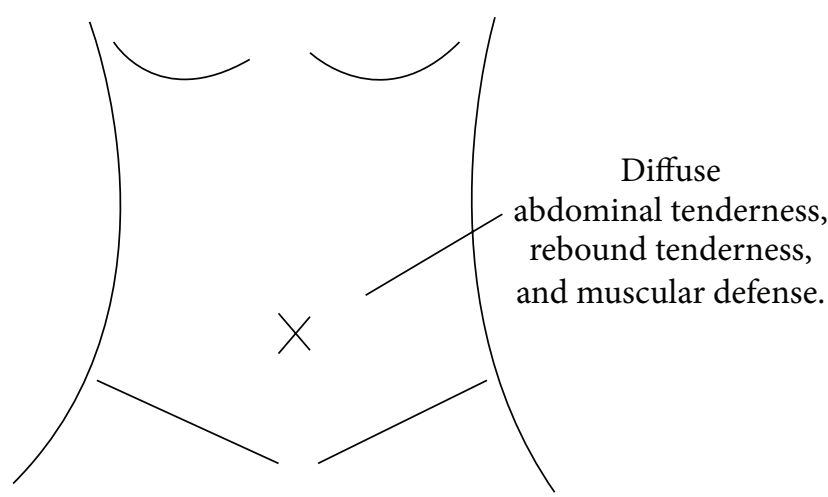

FIGURE 1

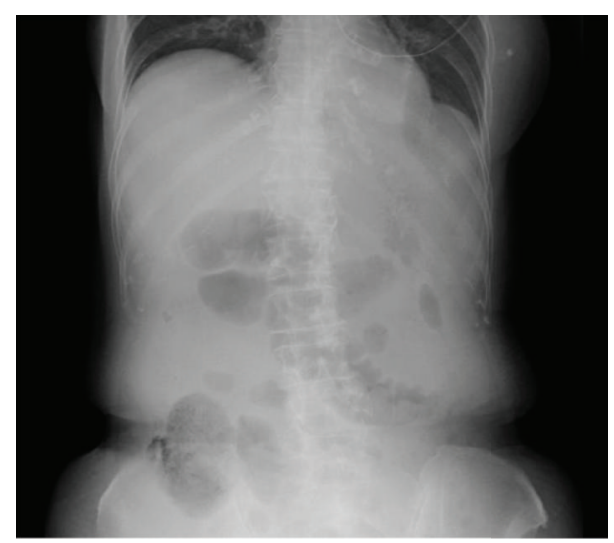

Figure 2: Plain abdominal X-ray film. No free intraperitoneal gas was observed.

3 days) and posttherapy (prednisolone tapering from $30 \mathrm{mg}$ daily) were initiated. During admission, she developed sudden epigastric pain and was referred to our department for consultation.

She was $147 \mathrm{~cm}$ tall and weighed $44 \mathrm{~kg}$, with a physical status of ASA 3. Her heart rate was $108 / \mathrm{min}$, blood pressure $158 / 92 \mathrm{mmHg}$, and respiratory rate $15 / \mathrm{min}$, with an $\mathrm{SpO}_{2}$ of $95 \%$. There was diffuse abdominal tenderness, rebound tenderness, and muscular defense (see Figure 1 and Table 1).

2.1. Plain Abdominal X-Ray Film (Supine). Bowel gas was deviated toward the midline, suggesting ascites. No free intraperitoneal gas was noted (Figure 2).

2.2. Plain Abdominal CT. Multiple sigmoid diverticula and a large amount of ascitic fluid were observed, with no evidence of bowel perforation (Figure 3).

This patient was an elderly woman who had nephrotic syndrome as an underlying condition and was taking steroids. Imaging studies failed to reveal any evidence of gastrointestinal perforation, presenting a diagnostic challenge as to whether or not to perform emergency surgery. Therefore, we performed abdominal paracentesis in the presence of a large amount of ascitic fluid.
TABLE 1: Blood test findings.

(a)

\begin{tabular}{lc}
\hline \multicolumn{3}{c}{ Complete blood count } \\
\hline WBC & $2.90 \times 10^{9} / \mathrm{L}$ \\
$\mathrm{RBC}$ & $4.20 \times 10^{12} / \mathrm{L}$ \\
$\mathrm{Hb}$ & $13.2 \mathrm{~g} / \mathrm{dL}$ \\
$\mathrm{Hct}$ & $39.6 \%$ \\
$\mathrm{Plt}$ & $120 \times 10^{9} / \mathrm{L}$ \\
\hline
\end{tabular}

(b)

\begin{tabular}{lc}
\hline \multicolumn{3}{c}{ Biochemistry } \\
\hline TP & $4.6 \mathrm{~g} / \mathrm{dL}$ \\
Tbil & $0.7 \mathrm{mg} / \mathrm{dL}$ \\
AST & $16 \mathrm{IU} / \mathrm{L}$ \\
ALT & $22 \mathrm{IU} / \mathrm{L}$ \\
CK & $40 \mathrm{IU} / \mathrm{L}$ \\
Amy & $33 \mathrm{IU} / \mathrm{L}$ \\
BUN & $24 \mathrm{mg} / \mathrm{dL}$ \\
Cre & $0.84 \mathrm{mg} / \mathrm{dL}$ \\
Na & $137 \mathrm{mmol} / \mathrm{L}$ \\
K & $4.0 \mathrm{mmol} / \mathrm{L}$ \\
CRP & $19.91 \mathrm{mg} / \mathrm{dL}$ \\
\hline
\end{tabular}

2.3. Ascitic Fluid. The ascitic fluid was milky, clear, odorless, with a protein content of $0.3 \mathrm{~g} / \mathrm{dL}$, an LDH level of $488 \mathrm{IU} / \mathrm{L}$, and a specific gravity of 1.010 (Figure 4).

Since the ascitic fluid had no smell of feces or that of the digestive system and was transudative, we considered that the presence of intestinal bacteria in the ascitic fluid, which should normally be sterile, would raise the possibility of gastrointestinal perforation. In addition, since it was at night when laboratory technicians were absent, a physician performed Gram staining.

2.4. Gram Staining. A small number of Gram-negative rods and a large number of leukocytes were seen (Figure 5).

Since Gram staining showed the presence of Gramnegative rods in the ascitic fluid, we strongly suspected digestive tract perforation and performed emergency surgery.

2.5. Intraoperative Findings. The abdomen was opened through a long midline abdominal incision. Ascitic fluid had accumulated in the peritoneal cavity. There were multiple sigmoid diverticula, one of which was perforated, and so Hartmann's procedure was performed with a diagnosis of perforated sigmoid diverticulum.

The operative time was $2 \mathrm{~h}$ and $43 \mathrm{~min}$. The amount of blood loss was $160 \mathrm{~mL}$. Two units of MAP, 10 units of PCs, and 2 units of FFP were transfused.

2.6. Resected Specimen. The resected sigmoid colon specimen measured $10 \times 8 \mathrm{~cm}$ and showed multiple stool-filled diverticula, one of which had a perforation, through which stool was visible (Figure 6). 


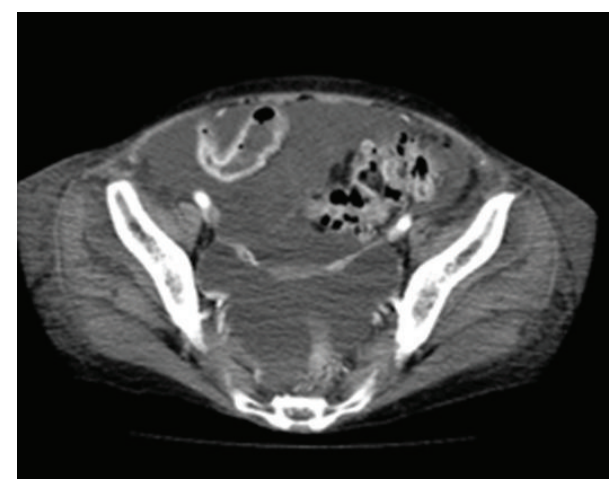

(a)

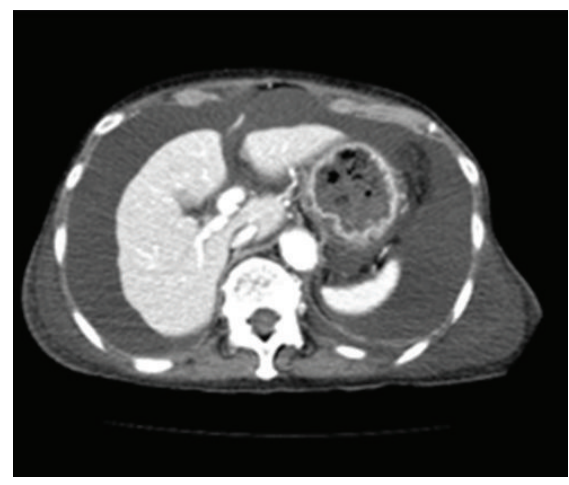

(b)

Figure 3: Plain abdominal CT. (a) Multiple sigmoid diverticula. (b) A large amount of ascitic fluid. No evidence of perforation was found.

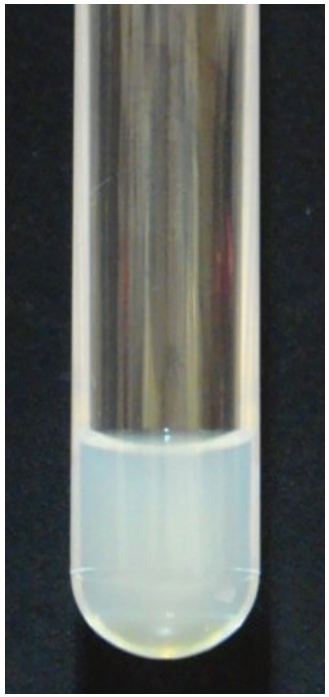

FIgURE 4: Ascitic fluid was milky, clear, and odorless.

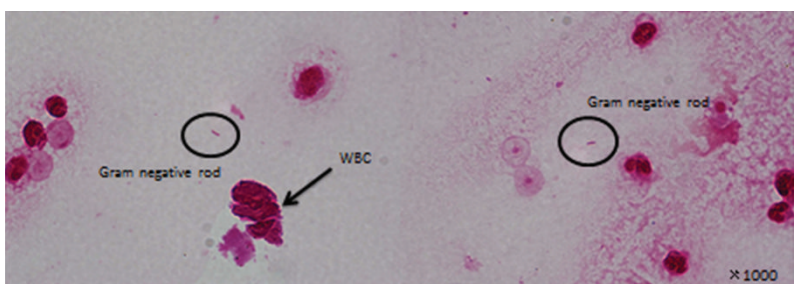

FIGURE 5: Rapid Gram staining of ascitic fluid. A small number of Gram-negative rods and a large number of leukocytes were seen.

2.7. Histopathological Findings. Multiple diverticula extending into the subserosa were present, and one of them was perforated. At this site, the diverticular wall was disrupted, and the surrounding tissue was infiltrated by neutrophils (Figure 7).

2.8. Microbiological Examinations of Ascitic Fluid. At a later date, the results of microbiological examinations of ascitic

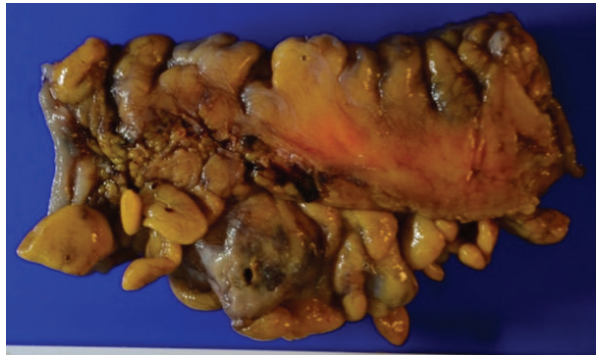

FIGURE 6: Resected specimen of the sigmoid colon. Multiple stoolfilled diverticula were found, one of which had a perforation, through which stool was visible.

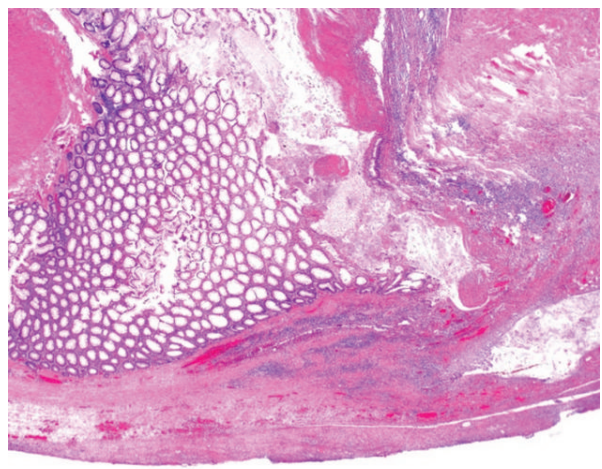

FIGURE 7: Histopathological findings. Diverticular perforation with neutrophilic infiltration of the surrounding tissue.

fluid were reported by the laboratory technician. Gramstained smears showed a small number of Gram-negative rods, Gram-positive cocci and rods, and +2 leukocytes. Cultures were positive for intestinal bacteria, including Bacteroides fragilis, being consistent with the results of the rapid diagnosis by Gram staining (Table 2).

2.9. Postoperative Course. The postoperative course was uneventful, and the patient was transferred to the general ward. Later, her hospital course became complicated by a 
TABLE 2: Bacteriological examination of ascitic fluid.

(a) Microbial smear examination

\begin{tabular}{lc}
\hline Name of bacteria & Number of bacterial cells \\
\hline Gram-negative rods & Small \\
Gram-positive cocci & Small \\
Gram-positive rods & Small \\
Leukocytes & $2+$ \\
\hline
\end{tabular}

(b) Bacterial cultures

\begin{tabular}{lc}
\hline Name of bacteria & Number of bacterial cells \\
\hline Bacteroides fragilis group & $1+$ \\
Propionibacterium sp. & Small \\
Micromonas micros & Small \\
Alpha-streptococci & Small \\
Bacillus sp. & Small \\
\hline
\end{tabular}

urinary tract infection, but she was discharged, after rehabilitation, on the 64th postoperative day.

\section{Discussion}

In the absence of imaging findings suggestive of gastrointestinal perforation, this patient presented a diagnostic challenge. However, since rapid Gram staining of ascitic fluid revealed Gram-negative rods, we strongly suspected gastrointestinal perforation, performed emergency surgery, and found a sigmoid colon perforation. Although it was at night, we performed rapid Gram staining, strongly suspected a gastrointestinal perforation, and were able to treat it surgically at an early stage.

Colon perforation easily causes septic shock due to intestinal bacteria, which leads to multiorgan failure with a poor prognosis if management is delayed. It is an important disease with a reported mortality rate of $17.6-29.4 \%$ [1-4]. Kuroda et al. [1] listed the following as prognostic factors for gastrointestinal perforation: (1) age $\geq 70$ years, (2) preoperative development of septic shock as a complication, (3) $\mathrm{WBC} \leq 4,000 / \mathrm{mm}^{3}$, (4) $\mathrm{BE} \leq-5$, (5) APACHE II score $\geq$ 20 , (6) generalized peritonitis, and (7) time to surgery $\geq 24$ hours. The time from onset to surgery is an important factor influencing the patient's prognosis. Therefore, it is necessary to make an early diagnosis of this condition using simple, rapid tests and start treatment.

Several radiologic modalities have a role in the diagnosis of gastrointestinal perforation, including plain X-ray film, ultrasonography, and computerized tomography [5]. CT is minimally invasive, and provides many objective data on the entire intraperitoneal contents within a short period of time $[6,7]$. It has been reported that the presence of free intraperitoneal air on CT indicates gastrointestinal perforation with a probability of $98.7 \%$ [8]. However, despite the presence of gastrointestinal perforation, as in the present case, CT did not reveal the presence of free intraperitoneal air in $25 \%$ [8], $83.3 \%$ [9], 64.3\% [10], and 80.5\% [11] of cases. In such cases, a decision must be made as to whether or not to perform surgery based on physical examination of the abdomen and other diagnostic means.

Gram staining can determine whether the patient has an infection within about 30 minutes after specimen collection. It is a very inexpensive test that can be performed using a glass slide and a small amount of staining solution [12], can estimate microorganisms based on their morphological characteristics, and is useful for the choice of subsequent treatments. In particular, if microorganisms are detected in normally sterile body fluids, such as blood, cerebrospinal fluid, pleural effusion, ascitic fluid, and joint fluid, they can be determined as pathogenic bacteria [12]. On the other hand, the disadvantage of Gram staining is the failure to detect a small number of microorganisms in a specimen: it is said that the specimen must contain more than 100,000 bacteria/mL to be deemed Gram positive. It is reportedly possible to microscopically examine as few as $1,000-10,000$ bacteria/mL of cerebrospinal, ascitic, or pleural fluid aspirate after centrifugation or sedimentation. Therefore, aspirates should be subjected to centrifugation or sedimentation before Gram staining. In addition, the results may vary according to the proficiency of the examiner, leading to a possible misdiagnosis [13]. For this reason, physicians should practice staining techniques and receive training in microscopy to aid in making an urgent diagnosis by Gram staining.

\section{Conclusion}

In this patient, CT findings were insufficient to suspect gastrointestinal perforation, but the detection of Gramnegative rods by Gram staining prompted us to perform emergency surgery. Gram staining should be performed in patients whose imaging shows no evidence of gastrointestinal perforation. If physicians themselves perform Gram staining, it may lead to prompt therapeutic intervention, making Gram staining an examination technique to be actively learned.

\section{Conflict of Interests}

There is no conflict of interests regarding the publication of this paper.

\section{References}

[1] H. Kuroda, A. Ito, M. Ido et al., "Criteria for assessment of severity and strategy in colorectal perforation," Journal of Abdominal Emergency Medicine, vol. 19, pp. 457-464, 1999 (Japanese).

[2] K.-I. Fukuda, O. Kinoshita, H. Nagata, A. Furutani, S. Nakashima, and M. Masuyama, "Prognostic factors in patients with colorectal perforation," Japanese Journal of Gastroenterological Surgery, vol. 41, no. 6, pp. 605-611, 2008 (Japanese).

[3] Y. Watanabe, Y. Nabeya, M. Matsuda et al., "Surgical treatment for perforation of the colon and rectum," Journal of Abdominal Emergency Medicine, vol. 19, pp. 473-479, 1999 (Japanese).

[4] C. Tono, H. Omori, Y. Inoue, H. Asahi, and K. Saito, "Diagnosis and treatment for perforation of the colon," Journal of Abdominal Emergency Medicine, vol. 19, pp. 465-471, 1999 (Japanese).

[5] S.-C. Chen, H.-P. Wang, W.-J. Chen et al., "Selective use of ultrasonography for the detection of pneumoperitoneum," Academic Emergency Medicine, vol. 9, no. 6, pp. 643-645, 2002. 
[6] R. Zissin, M. Hertz, A. Osadchy, E. Even-Sapir, and G. Gayer, "Abdominal CT findings in nontraumatic colorectal perforation," European Journal of Radiology, vol. 65, no. 1, pp. 125-132, 2008.

[7] A. Furukawa, M. Sakoda, M. Yamasaki et al., "Gastrointestinal tract perforation: CT diagnosis of presence, site, and cause," Abdominal Imaging, vol. 30, no. 5, pp. 524-534, 2005.

[8] T. Kyokane, S. Iyomasa, N. Sawasaki et al., "The utility of computed tomography on diagnosis of the alimentary tract perforation," Japanese Journal of Gastroenterological Surgery, vol. 42, no. 6, pp. 622-631, 2009 (Japanese).

[9] K. Sugimoto, S. Ono, S. Ishiyama et al., "Study of predictive factors regarding prognoses and post-operative hospitalization periods of patients with colorectal perforation," Journal of Abdominal Emergency Medicine, vol. 31, pp. 19-27, 2011 (Japanese).

[10] V. Maniatis, H. Chryssikopoulos, A. Roussakis et al., "Perforation of the alimentary tract: evaluation with computed tomography," Abdominal Imaging, vol. 25, no. 4, pp. 373-379, 2000 (Japanese).

[11] M. Tabata, K. Sakoda, J. Mizouchi, H. Sakamoto, and M. Osako, "Clinical study of free perforation of the colorectum," Journal of Abdominal Emergency Medicine, vol. 19, pp. 429-435, 1999 (Japanese).

[12] K. Yamanaka, "Reviews for gram stain," The Japanese Journal of Clinical Pathology, vol. 50, no. 5, pp. 449-454, 2002 (Japanese).

[13] T. Oguri, "Kanzya kentai no gram sensyoku rinsho to biseibutsu," Clinical Microbiology, vol. 22, pp. 635-642, 1995. 


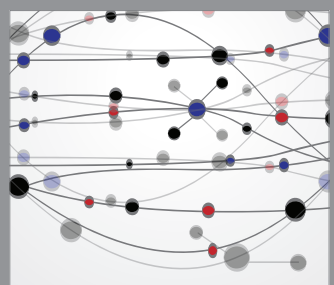

The Scientific World Journal
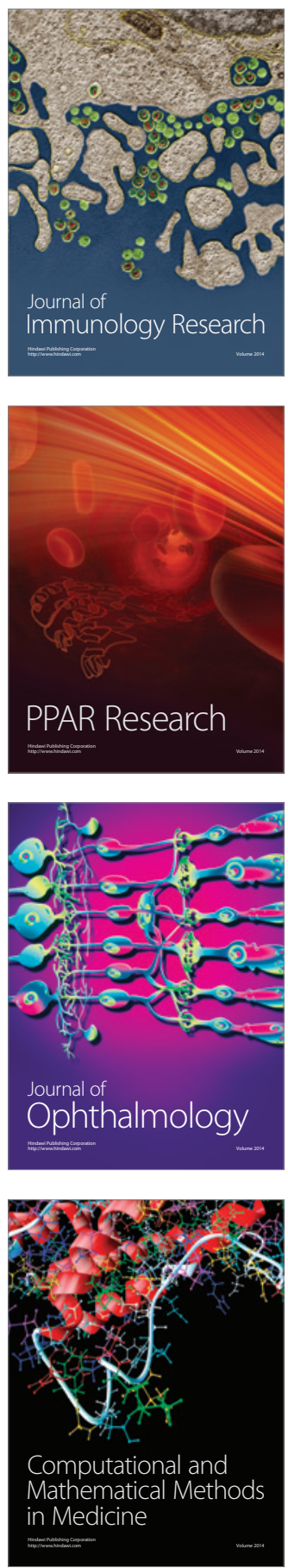

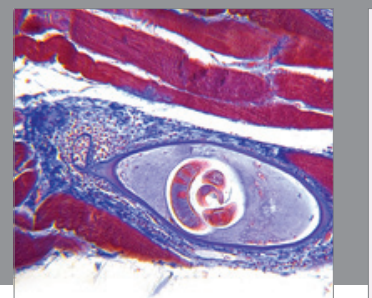

Gastroenterology

Research and Practice
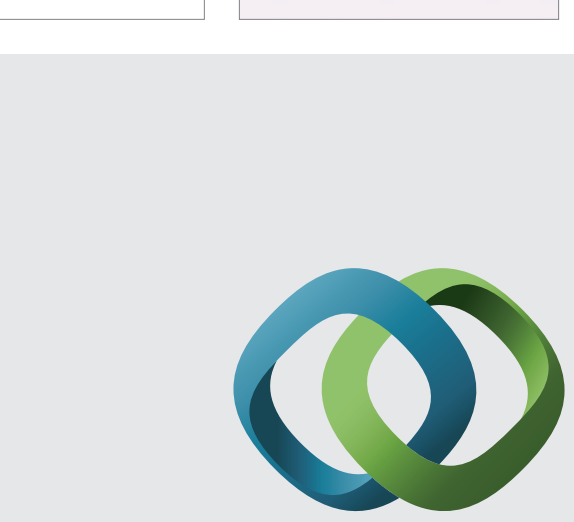

\section{Hindawi}

Submit your manuscripts at

http://www.hindawi.com
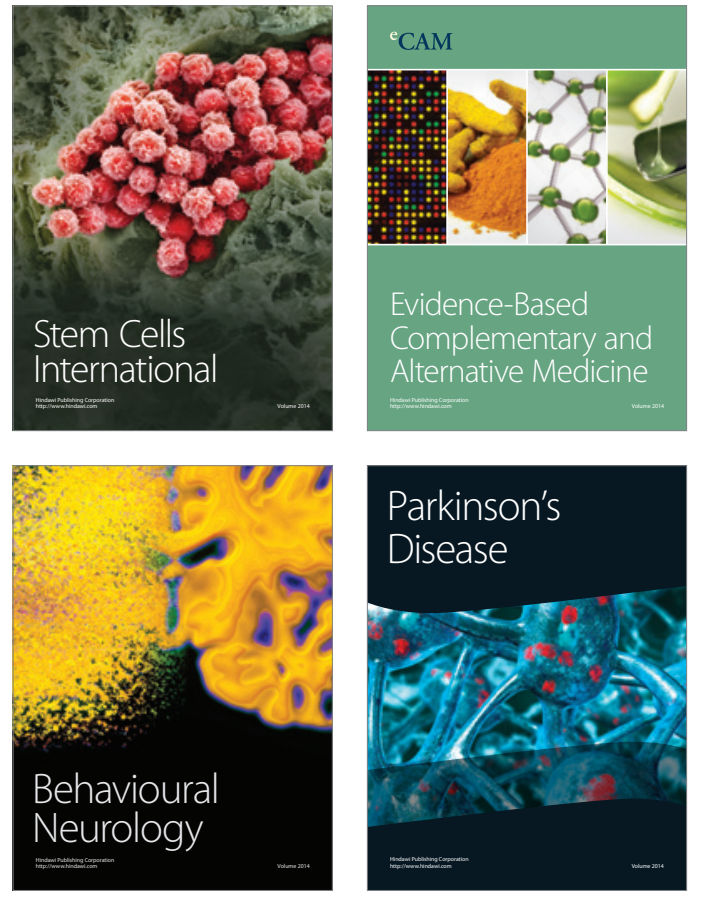
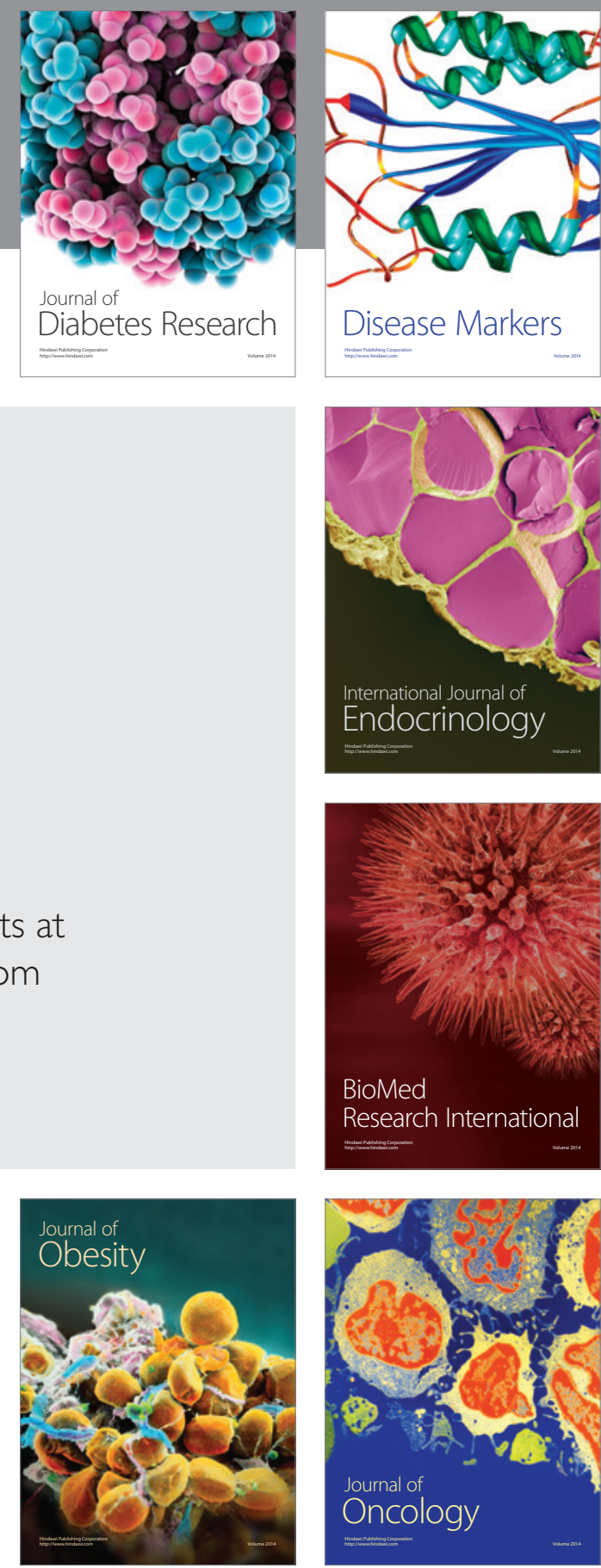

Disease Markers
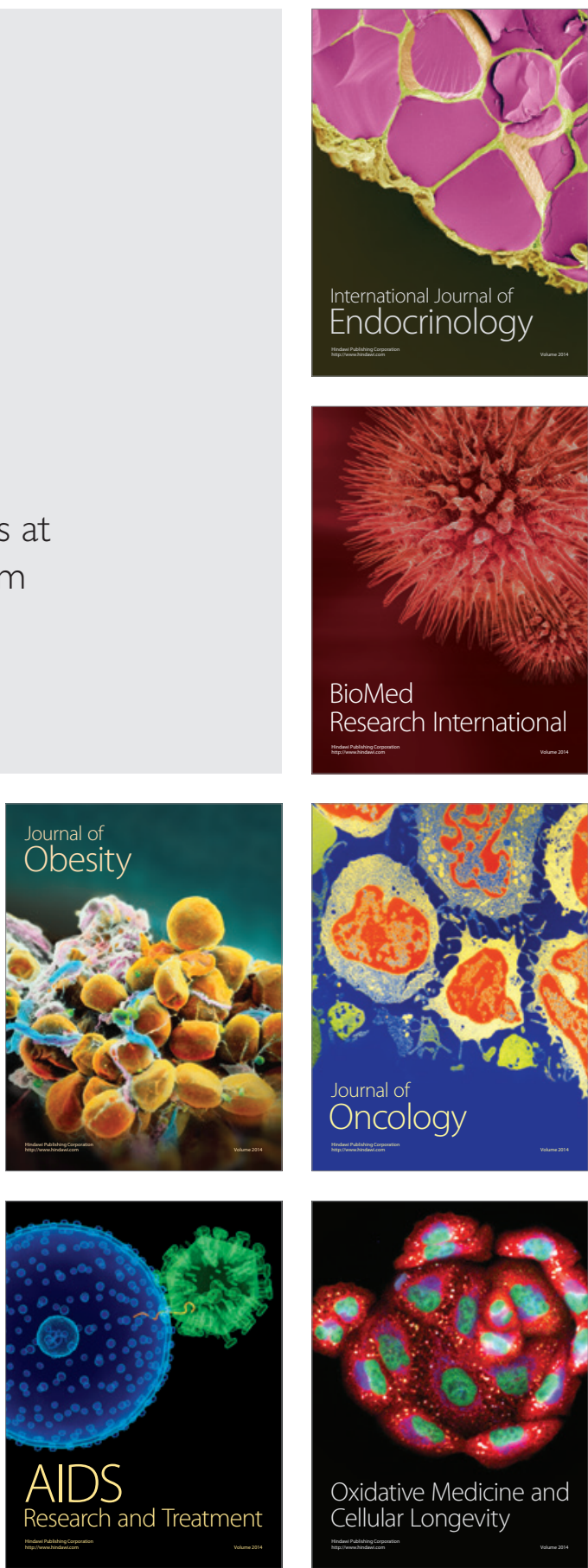